\title{
ÍNDICE DE VEGETAÇÃO POR DIFERENÇA NORMALIZADA E PRODUTIVIDADE DE CULTIVARES DE TRIGO SUBMETIDAS A DOSES DE NITROGÊNIO
}

\author{
Adriano Luiz Lodi Rissini ${ }^{(1)}$, Jackson Kawakami ${ }^{(2)}$ e Aline Marques Genú( ${ }^{(2) *}$ \\ (1) Universidade Estadual do Centro-Oeste, Departamento de Agronomia, Programa de Pós-graduação em Agronomia, Guarapuava, \\ Paraná, Brasil. \\ (2) Universidade Estadual do Centro-Oeste, Departamento de Agronomia, Guarapuava, Paraná, Brasil. \\ * Autor correspondente. \\ E-mail: agenu@unicentro.br
}

\begin{abstract}
RESUMO
O manejo da adubação nitrogenada na cultura do trigo tem se evidenciado fator limitante à produtividade da cultura na Região Sul do Brasil, e a utilização de novas técnicas como o índice de vegetação por diferença normalizada (NDVI) pode auxiliar na estimativa do potencial produtivo da cultura e, consequentemente, na determinação da adubação nitrogenada. $O$ objetivo deste trabalho foi avaliar o uso do NDVI, obtido por sensores passivo e ativo, como ferramenta para estimar a produtividade de grãos de cultivares de trigo submetidas a doses de nitrogênio. $O$ experimento foi conduzido em Guarapuava, $P R$, em blocos ao acaso, seguindo o esquema fatorial $4 \times 5$, com quatro cultivares e cinco doses de $\mathrm{N}$. As avaliações realizadas compreenderam leituras de NDVI, matéria seca (MS) de parte aérea, nos estádios fenológicos de afilhamento, emborrachamento e florescimento, teor foliar de $\mathrm{N}$ e produtividade de grãos. Nas leituras de NDVI, constataram-se diferenças significativas entre cultivares em todos os estádios fenológicos e doses, assim como entre as doses e os estádios fenológicos, para ambos os sensores. Verificaram-se efeito da MS e do teor foliar de $\mathrm{N}$ nas leituras de NDVI, sendo possível gerar modelos para a estimativa desses atributos com base no NDVI e, ainda, estimar a produtividade no início de desenvolvimento da cultura.
\end{abstract}

Palavras-chave: adubação nitrogenada, sensoriamento remoto, Triticum aestivum. 


\title{
ABSTRACT: NORMALIZED DIFFERENCE VEGETATION INDEX AND YIELD OF WHEAT CULTIVARS UNDER DIFFERENT APPLICATION RATES OF NITROGEN
}

\begin{abstract}
The management of nitrogen fertilization in wheat has proven to be a limiting factor for grain yield in the southern region of Brazil, and new techniques like the normalized difference vegetation index (NDVI) can be useful for estimating crop yield potential and, consequently, for determining $N$ fertilization requirements. The objective of this study was to evaluate the use of NDVI, obtained through active and passive sensors, as a tool for estimating the grain yield of wheat cultivars under different $N$ application rates. The experiment was conducted in Guarapuava, PR, Brazil, using a randomized block design and a $4 \times 5$ factorial structure, with four cultivars and five $N$ rates. Evaluations consisted of NDVI readings; shoot dry matter (DM) at the phenological stages of tillering, booting, and flowering; leaf $N$ concentration, and grain yield. For both sensors, significant differences in NDVI readings were found among cultivars in all phenological stages and at all $N$ rates, as well as among $N$ rates and phenological stages. The results revealed $D M$ and leaf $N$ effects on NDVI readings, and the possibility of generating models to estimate these parameters based on NDVI readings and also for estimating grain yield at an early crop development stage.
\end{abstract}

Keywords: nitrogen fertilization, remote sensing, Triticum aestivum.

\section{INTRODUÇÃO}

O trigo (Triticum aestivum L.) é cultivado aproximadamente em 126 países, e, dentre os cereais cultivados, ele se destaca como um dos mais produzidos, atingindo cerca de 716 milhões de toneladas, com produtividade média de 3,3 $\mathrm{Mg} \mathrm{ha}^{-1}$ na safra de 2012/2013 (FAO, 2015). Os maiores produtores mundiais são China, Índia, EUA, Rússia e França (FAO, 2015). No Brasil, a cultura tem grande importância econômica para a Região Sul do país, onde especificamente no município de Guarapuava, Estado do Paraná, $9^{\circ}$ maior município produtor na safra 2012/2013, atingem-se altas produtividades médias, da ordem de 3,9 $\mathrm{Mg} \mathrm{ha}^{-1}$ (IBGE, 2015).

O lançamento de inúmeras cultivares de elevado potencial produtivo leva ao aumento da adubação, principalmente a nitrogenada, visto que $\mathrm{N}$ é o nutriente mais requerido e mais exportado. Entre os elementos componentes da adubação, o $\mathrm{N}$ é o que mais tem limitado a produtividade da cultura do trigo (Zagonel et al., 2002).

$\mathrm{O} \mathrm{N}$ é um dos nutrientes mais difíceis de manejar nos solos de regiões subtropicais, em razão da alta instabilidade deste no solo, uma vez que está sujeito a grande número de reações (Ernani, 2003). Geralmente, a dose a ser aplicada para a cultura em questão corresponde ao resultado de uma série de estudos de calibração e é determinada pelo resultado produtivo da cultura (Gastal e Lemaire, 2002). Entretanto, essas calibrações são geralmente realizadas em locais e cultivares específicos, sendo então generalizadas para uma ampla região e as mais diversas cultivares, o que nem sempre permite a exploração máxima do potencial produtivo da região.
O máximo potencial produtivo para as culturas agrícolas pode ser estimado em qualquer estádio de seu desenvolvimento; para o trigo, essa estimativa é definida no período de emissão da $6^{a}$ folha do colmo principal, época em que se define o número máximo de afilhos emitidos pela planta. É, também, nessa época, que se tem o período crítico para aplicação de N, uma vez que é nesse momento que a planta iniciará a máxima exigência por esse nutriente (Grohs et al., 2009).

Nesse sentido, técnicas de sensoriamento remoto podem ser utilizadas com o objetivo de gerar informações adicionais no momento da recomendação da adubação nitrogenada. Dados da resposta espectral do dossel da cultura, durante seu desenvolvimento, caracterizados pelo processo de reflectância de ondas eletromagnéticas incidentes, podem ser utilizados como indicadores indiretos do status nutricional da cultura, bem como do potencial produtivo (Serrano et al., 2000). Esses dados são obtidos via sensores, classificados em ativos (quando possuem fonte própria de energia que incida sob as plantas) e passivos (quando dependem da radiação solar como fonte de energia).

Existem dois comprimentos de onda que apresentam relação direta com variáveis na planta, indicadores do potencial produtivo: ondas do espectro do vermelho, cuja reflectância é menor em condições de maior quantidade de clorofila (Tucker, 1979); e ondas do espectro do infravermelho, cuja reflectância é maior quanto maior for o acúmulo de massa de matéria seca (MS) da planta. $\mathrm{O}$ teor de clorofila da folha, assim como a produção de MS, se correlaciona positivamente com doses de $\mathrm{N}$ e com a produtividade (Schadchina e Dmitrieva, 1995).

Para minimizar a variabilidade causada por fatores externos nos dados obtidos de reflectância, 
utilizam-se índices de vegetação, que são sensíveis à biomassa verde de uma comunidade de plantas e, portanto, à quantidade de clorofila por unidade de área (Ponzoni, 2001).

O índice de vegetação por diferença normalizada, conhecido como NDVI (Rouse et al., 1973), utiliza dados de reflectância do vermelho e infravermelho; os valores desses dados vão de uma escala de -1 a 1 . Quanto maiores os valores do NDVI, maiores as diferenças entre a reflectância do infravermelho com o vermelho, o que indica maior quantidade de clorofila e MS e, consequentemente, maior o potencial produtivo da planta.

Com base nesse contexto, este trabalho teve por objetivo avaliar o uso do NDVI, obtido por meio de sensor ativo e passivo, como ferramenta para estimar a produtividade de cultivares de trigo submetidas a doses de $\mathrm{N}$ em três estádios de desenvolvimento na região centro-sul do Paraná, partindo-se da hipótese de que leituras da resposta espectral da cultura, através do NDVI, são diretamente influenciados pela quantidade de $\mathrm{N}$ disponibilizado e absorvido pela planta, uma vez que este interfere diretamente no teor de clorofila e consequentemente nas características espectrais da mesma, bem como, nas variáveis que compõem a produtividade final.

\section{MATERIAL E MÉTODOS}

O trabalho foi realizado no município de Guarapuava, região centro-sul do Estado do Paraná, durante a safra de inverno de 2009. O município está situado no terceiro planalto paranaense a $1.100 \mathrm{~m}$ de altitude. A precipitação pluvial e temperatura média anual são de $2.002 \mathrm{~mm}$ e $16,8{ }^{\circ} \mathrm{C}$, respectivamente, e o clima da região é caracterizado como subtropical úmido sem estações secas e com geadas severas frequentes.

O experimento foi implantado em área experimental, de topografia plana. O soloé classificado como Latossolo Bruno, de textura muito argilosa (Embrapa, 2006). Para caracterizar a fertilidade do solo (Pavan et al., 1992), realizou-se amostragem com 30 pontos (subamostra), estratificada nas profundidades de 0,0-0,1 e 0,1-0,2 m, cujos valores foram, respectivamente: $\mathrm{pH}\left(\mathrm{CaCl}_{2}\right): 5,1 \mathrm{e} 4,4 ; \mathrm{Al}^{3+}$ : 0,00 e $0,46 \mathrm{cmol}_{\mathrm{c}} \mathrm{dm}^{-3} ; \mathrm{Ca}^{2+}: 7,03$ e $3,79 \mathrm{cmol}_{\mathrm{c}} \mathrm{dm}^{-3}$; $\mathrm{Mg}^{2+}: 2,74$ e $1,14 \mathrm{cmol}_{\mathrm{c}} \mathrm{dm}^{-3} ; \mathrm{K}^{+}: 0,80$ e $0,45 \mathrm{cmol}_{\mathrm{c}} \mathrm{dm}^{-3}$; P: 20,90 e 14,90 $\mathrm{mg} \mathrm{dm}^{-3}$; Carbono orgânico: 43,64 e $34,68 \mathrm{~g} \mathrm{dm}^{-3}$; e V: 59,48 e $30,73 \%$.

As cultivares de trigo utilizadas foram 'Quartzo', 'Campeiro', 'Tangara' e 'CD 115'. A semeadura foi realizada no dia 03/07/2009, com espaçamento de $0,17 \mathrm{~m}$, para uma população esperada de 330 plantas por $\mathrm{m}^{2}$, com análise de $91 \%-87 \%-36 \mathrm{~g}$;
$91 \%-81 \%-29$ g; $92 \%-90 \%-38,2$ g; e $90 \%$ $82 \%-31 \mathrm{~g}$ de poder germinativo, vigor e peso de 1.000 sementes, respectivamente.

A adubação de base foi de $90 \mathrm{~kg} \mathrm{ha}^{-1}$ de $\mathrm{P}_{2} \mathrm{O}_{5} \mathrm{e}$ $60 \mathrm{~kg} \mathrm{ha}^{-1}$ de $\mathrm{K}_{2} \mathrm{O}$, na forma de superfosfato triplo e cloreto de potássio, respectivamente. As doses de $\mathrm{N}$ foram aplicadas no sulco da semeadura com doses de 30,60, 90 e $120 \mathrm{~kg} \mathrm{ha}^{-1}$, na forma de ureia, mantendo-se uma testemunha sem aplicação de N. As doses completas de $\mathrm{N}$ foram aplicadas na base, com o intuito de se verificar o seu efeito na resposta do NDVI logo no início do ciclo. O delineamento experimental foi de blocos ao acaso num esquema fatorial de $4 \times 5$ (cultivares $\times$ doses) em quatro repetições, com parcelas de $3 \times 5 \mathrm{~m}$.

As leituras de NDVI foram realizadas em três épocas durante o desenvolvimento da cultura, sendo caracterizadas pela escala fenológica de Feeks e Large (Large, 1954): Feeks 3 (afilhamento), Feeks 8 (início do emborrachamento) e Feeks 10.5.1 (início do florescimento) nos dias 15/08, 15/09 e 23/10 de 2009, respectivamente. Nessas mesmas datas, realizou-se a coleta de plantas em 0,50 m linear de cada parcela, sendo essas submetidas à secagem em estufa a $65{ }^{\circ} \mathrm{C}$ para se determinar a massa de matéria seca (MS) da parte aérea das plantas. Para definir o teor foliar de N, coletaram-se 50 folhas bandeiras de cada parcela em Feeks 10.5.1, de acordo com Cantarella et al. (1997). A produtividade final de grãos foi determinada pela colheita nas 12 linhas centrais em 3 m linear para cada parcela, sendo a massa de grãos corrigida para $13 \%$ de umidade.

O sensor ótico ativo utilizado foi o Crop Circle ACS 210. É um sensor portátil que tem como emissor de luz um vetor de led's policromáticos modulados que emitem feixes de luz num comprimento de onda de 650 a $850 \mathrm{~nm}$, o que compreende a faixa do vermelho e infravermelho próximo. Para a fotodeteç̧ão, possui dois detectores de luz, um na faixa de 400 a $680 \mathrm{~nm}$ e outro na de 800 a $1.100 \mathrm{~nm}$. Os dados de saída correspondem ao Índice de Vegetação por Diferença Normalizada (NDVI). Os dados foram coletados a uma distância de 0,8 a 1,0 m entre o sensor e o alvo.

Para o sensor ótico passivo, foi utilizada uma câmera fotográfica multiespectral, marca Fuji, 8 bits, registro de quatro bandas (vermelho, infravermelho próximo, verde e azul), em 256 níveis de cinza. Para a captura das imagens, utilizou-se uma aeronave de pequeno porte, que sobrevoou as parcelas a uma altitude média de $150 \mathrm{~m}$, entre 10 e $14 \mathrm{~h}$.

Dados de NDVI foram analisados, comparandose as cultivares dentro de cada dose, bem como leituras de NDVI, comparando-se sensores dentro de cada cultivar. Os dados foram submetidos à Anova, seguido do teste de Tukey para comparar as médias a $5 \%$. Realizou-se a análise de regressão, ajustando-se 
os modelos para NDVI e MS, NDVI e teor foliar de $\mathrm{N}$, MS e produtividade e NDVI e produtividade. $\mathrm{O}$ modelo de regressão utilizado foi o que apresentou maior coeficiente de determinação $\left(\mathrm{R}^{2}\right)$, submetido ao teste $\mathrm{F}$ a $1 \%$.

\section{RESULTADOS E DISCUSSÃO}

\section{Índice de Vegetação por Diferença Normalizada (NDVI)}

Houve diferença significativa do NDVI para as cultivares, as doses, os estádios e as interações, para ambos os sensores avaliados, com exceção da interação cultivar $\times$ estádio $\times$ dose para o sensor ativo (Quadro 1). O comportamento do NDVI no sensor passivo variou para as cultivares durante o ciclo de desenvolvimento e para as doses de $\mathrm{N}$ utilizadas; no entanto, esse comportamento foi distinto para cada cultivar (Quadro 2). A cultivar Campeiro, sem N, apresentou menor NDVI em Feeks 3 e depois valores estatisticamente iguais para Feeks 8 e 10.5.1, enquanto nas demais doses o NDVI foi diferente em cada estádio fenológico, sendo mais elevado em Feeks 8. Para a 'Quartzo', só houve diferença nos três estádios para a dose 0 de $\mathrm{N}$; nas demais doses, houve diferença apenas em Feeks 3. Para a 'CD115', a diferença entre os três estádios ocorreu nas doses 30 e $60 \mathrm{~g} \mathrm{~kg}^{-1}$ de N; na 'Tangara', para todas as doses. O NDVI foi diferente em cada Feeks, sendo maior em Feeks 8.

Para o sensor ativo (Quadro 2), os resultados do NDVI para as cultivares durante o ciclo de desenvolvimento, nas doses de $\mathrm{N}$ utilizadas, foram diferentes; para as cultivares 'Tangara' e 'CD115' houve diferença nos três estádios avaliados para todas as doses, enquanto a 'Campeiro' não apresentou essa diferença, apenas para dose 0; e a
'Quartzo' evidenciou diferença entre os três estádios nas doses 60 e $90 \mathrm{~g} \mathrm{~kg}^{-1}$ de $\mathrm{N}$.

De forma semelhante, o comportamento das cultivares em relação às doses para cada estádio fenológico é distinto, especialmente entre 0 e $30 \mathrm{~g} \mathrm{~kg}^{-1}$ de $\mathrm{N}$, tanto para o sensor passivo quanto ativo (Quadro 2). Esses resultados demonstraram que cada cultivar apresenta um comportamento espectral distinto, o que está relacionado com suas características morfológicas e de desenvolvimento inicial da cultura e, ainda, indicaram que o aumento das doses, entre 60 e $120 \mathrm{~g} \mathrm{~kg}^{-1}$, não influenciou significativamente o NDVI, nos estádios avaliados para a maioria das cultivares, justificando a necessidade de se avaliarem as cultivares em separado para estimar a produtividade e recomendar a adubação nitrogenada.

Utilizando o sensor passivo, houve diferenças significativas de NDVI para as cultivares, em razão das doses para cada estádio fenológico, em Feeks 3 para as doses 30, 60, 90 e $120 \mathrm{~kg} \mathrm{ha}^{-1}$ de $\mathrm{N}$; em Feeks 8, para as doses 0, 30 e $90 \mathrm{~kg} \mathrm{ha}^{-1}$ de $\mathrm{N}$; e em Feeks 10.5.1, nas doses 60 e $90 \mathrm{~kg} \mathrm{ha}^{-1} \mathrm{de}$ $\mathrm{N}$ (Figura 1). Já com o sensor ativo, as diferenças de NDVI foram significativas em Feeks 3 para as doses 0, 60, 90 e $120 \mathrm{~kg} \mathrm{ha}^{-1}$ de N; em Feeks 8, para as doses 0, 30, 90 e $120 \mathrm{~kg} \mathrm{ha}^{-1}$ de $\mathrm{N}$; e em Feeks 10.5.1, nas doses 0, 30 e $90 \mathrm{~kg} \mathrm{ha}^{-1}$ de N (Figura 2).

Nenhuma cultivar apresentou acamamento que pudesse comprometer os resultados de NDVI, mesmo nas maiores doses de N. No entanto, características como ângulo foliar e precocidade no desenvolvimento inicial fazem com que a cultivar Tangara se destaque frente às demais, respondendo com maiores valores de NDVI, quando comparado com as demais cultivares em Feeks 3, principalmente, mas também em Feeks 8, com ambos os sensores, sendo esse resultado menos evidente no estádio fenológico 10.5.1.

Quadro 1. Análise de variância para Índice de Vegetação por Diferença Normalizada (NDVI) obtido por sensores passivo (SP) e ativo (SA)

\begin{tabular}{lccc}
\hline \multirow{2}{*}{ Fonte de variação } & Grau de liberdade & Quadrado Médio \\
\cline { 3 - 4 } & & NDVI_SP & NDVI_SA \\
\hline Cultivar & 3 & $0,014904^{* *}$ & $0,004689^{* *}$ \\
Dose & 4 & $0,163957^{* *}$ & $1,9979758^{* *}$ \\
Estádio & 2 & $1,841812^{* *}$ & $0,000628^{* * *}$ \\
Cultivar × Dose & 12 & $0,001285^{* *}$ & $0,002645^{* *}$ \\
Cultivar $\times$ Época & 6 & $0,008106^{* *}$ & $0,001649^{* *}$ \\
Dose $\times$ Época & 8 & $0,002419^{* *}$ & $0,000272^{\mathrm{ns}}$ \\
Cultivar $\times$ Época $\times$ Dose & 24 & $0,001284^{* *}$ & 0,000219 \\
Erro & 180 & 0,000556 & \\
Total Corrigido & 239 & & \\
\hline
\end{tabular}

**: significativo a $1 \%$ pelo teste $\mathrm{F}$; ${ }^{\text {ns: }}$ não significativo. 
Quadro 2. Índice de vegetação por diferença normalizada (NDVI) obtido por sensores passivo e ativo, das cultivares de trigo nos diferentes estádios fenológicos e doses de nitrogênio

\begin{tabular}{|c|c|c|c|c|c|c|}
\hline \multirow{2}{*}{ Cultivar } & \multirow{2}{*}{ Estádio $^{(1)}$} & \multicolumn{5}{|c|}{ Dose de $N$} \\
\hline & & $0 \mathrm{~kg} \mathrm{ha}^{-1}$ & $30 \mathrm{~kg} \mathrm{ha}^{-1}$ & $60 \mathrm{~kg} \mathrm{ha}^{-1}$ & $90 \mathrm{~kg} \mathrm{ha}^{-1}$ & $120 \mathrm{~kg} \mathrm{ha}^{-1}$ \\
\hline & & & & Passivo & & \\
\hline \multirow[t]{3}{*}{ Campeiro } & 3 & $0,032 \mathrm{aA}^{(2)}$ & $0,115 \mathrm{aB}$ & $0,147 \mathrm{aBC}$ & $0,187 \mathrm{aC}$ & $0,155 \mathrm{aBC}$ \\
\hline & 8 & $0,285 \mathrm{bA}$ & $0,417 \mathrm{cB}$ & $0,457 \mathrm{cBC}$ & $0,452 \mathrm{cBC}$ & $0,470 \mathrm{cC}$ \\
\hline & 10.5 .1 & $0,280 \mathrm{bA}$ & $0,340 \mathrm{bB}$ & $0,382 \mathrm{bC}$ & $0,382 \mathrm{bBC}$ & $0,412 \mathrm{bC}$ \\
\hline \multirow[t]{3}{*}{ Quartzo } & 3 & $0,035 \mathrm{aA}$ & $0,102 \mathrm{aB}$ & $0,147 \mathrm{aB}$ & $0,142 \mathrm{aB}$ & $0,147 \mathrm{aB}$ \\
\hline & 8 & $0,255 \mathrm{bA}$ & $0,375 \mathrm{bB}$ & $0,452 \mathrm{bC}$ & $0,447 \mathrm{bC}$ & $0,465 \mathrm{bC}$ \\
\hline & 10.5 .1 & $0,307 \mathrm{cA}$ & $0,357 \mathrm{bB}$ & $0,420 \mathrm{bC}$ & $0,430 \mathrm{bC}$ & $0,447 \mathrm{bC}$ \\
\hline \multirow[t]{3}{*}{ CD115 } & 3 & $0,055 \mathrm{aA}$ & $0,125 \mathrm{aB}$ & $0,167 \mathrm{aB}$ & $0,155 \mathrm{aB}$ & $0,150 \mathrm{aB}$ \\
\hline & 8 & $0,325 \mathrm{bA}$ & $0,417 \mathrm{cB}$ & $0,440 \mathrm{cb}$ & $0,450 \mathrm{bB}$ & $0,460 \mathrm{bB}$ \\
\hline & 10.5 .1 & $0,310 \mathrm{bA}$ & $0,360 \mathrm{bB}$ & $0,382 \mathrm{bBC}$ & $0,422 \mathrm{bCD}$ & $0,442 \mathrm{bD}$ \\
\hline \multirow[t]{4}{*}{ Tangara } & 3 & $0,065 \mathrm{aA}$ & $0,145 \mathrm{aB}$ & $0,235 \mathrm{aC}$ & $0,232 \mathrm{aC}$ & $0,242 \mathrm{aC}$ \\
\hline & 8 & $0,370 \mathrm{cA}$ & $0,427 \mathrm{cB}$ & $0,477 \mathrm{cC}$ & $0,500 \mathrm{cC}$ & $0,475 \mathrm{cC}$ \\
\hline & 10.5 .1 & $0,305 \mathrm{bA}$ & $0,330 \mathrm{bA}$ & $0,377 \mathrm{bB}$ & $0,435 \mathrm{bC}$ & $0,412 \mathrm{bC}$ \\
\hline & & & & Ativo & & \\
\hline \multirow[t]{3}{*}{ Campeiro } & 3 & $0,387 \mathrm{aA}$ & $0,430 \mathrm{aB}$ & $0,445 \mathrm{aBC}$ & $0,442 \mathrm{aC}$ & $0,425 \mathrm{aBC}$ \\
\hline & 8 & $0,655 \mathrm{bA}$ & $0,712 \mathrm{cB}$ & $0,747 \mathrm{cBC}$ & $0,752 \mathrm{cBC}$ & $0,755 \mathrm{cC}$ \\
\hline & 10.5 .1 & $0,637 \mathrm{bA}$ & $0,652 \mathrm{bB}$ & $0,690 \mathrm{bC}$ & $0,692 \mathrm{bBC}$ & $0,705 \mathrm{bC}$ \\
\hline \multirow[t]{3}{*}{ Quartzo } & 3 & $0,405 \mathrm{aA}$ & $0,435 \mathrm{aB}$ & $0,440 \mathrm{aB}$ & $0,440 \mathrm{aB}$ & $0,432 \mathrm{aB}$ \\
\hline & 8 & $0,642 \mathrm{bA}$ & $0,697 \mathrm{bB}$ & $0,742 \mathrm{cC}$ & $0,740 \mathrm{cC}$ & $0,742 \mathrm{bC}$ \\
\hline & 10.5 .1 & $0,665 \mathrm{bA}$ & $0,680 \mathrm{bB}$ & $0,705 \mathrm{bC}$ & $0,715 \mathrm{bC}$ & $0,722 \mathrm{bC}$ \\
\hline \multirow[t]{3}{*}{ CD115 } & 3 & $0,392 \mathrm{aA}$ & $0,432 \mathrm{aB}$ & $0,447 \mathrm{aB}$ & $0,445 \mathrm{aB}$ & $0,447 \mathrm{aB}$ \\
\hline & 8 & $0,697 \mathrm{cA}$ & $0,732 \mathrm{cB}$ & $0,750 \mathrm{cB}$ & $0,755 \mathrm{cB}$ & $0,765 \mathrm{cB}$ \\
\hline & 10.5 .1 & $0,692 \mathrm{bA}$ & $0,667 \mathrm{bB}$ & $0,690 \mathrm{bBC}$ & $0,705 \mathrm{bCD}$ & $0,712 \mathrm{bD}$ \\
\hline \multirow[t]{3}{*}{ Tangara } & 3 & $0,417 \mathrm{aA}$ & $0,427 \mathrm{aB}$ & $0,475 \mathrm{aC}$ & $0,489 \mathrm{aC}$ & $0,492 \mathrm{aC}$ \\
\hline & 8 & $0,695 \mathrm{cA}$ & $0,722 \mathrm{cB}$ & $0,757 \mathrm{cC}$ & $0,770 \mathrm{cC}$ & $0,777 \mathrm{cC}$ \\
\hline & 10.5 .1 & $0,647 \mathrm{bA}$ & $0,650 \mathrm{bA}$ & $0,685 \mathrm{bB}$ & $0,720 \mathrm{bC}$ & $0,722 \mathrm{bBC}$ \\
\hline
\end{tabular}

(1) Estádio fenológico: Feeks 3, Feeks 8 e Feeks 10.5.1; ${ }^{(2)}$ Letras minúsculas na coluna comparam os estádios fenológicos para a mesma cultivar e dose de $\mathrm{N}$, e letras maiúsculas comparam as doses de $\mathrm{N}$ para a mesma cultivar e estádio fenológico, pelo teste de Tukey a $5 \%$.

Em estudo sobre a variação espectral de 82 genótipos de trigo, entre o estádio de emborrachamento e ponto de colheita, Hatfield (1981) observou variabilidade na resposta espectral dos genótipos, concluindo que as diferenças estão associadas não apenas às variáveis agronômicas, como índice de área foliar (IAF) ou acúmulo de biomassa, mas também à alta dependência da geometria de incidência da radiação e sua interação com as características da arquitetura dos dosséis vegetativos.

Diferenças entre cultivares de trigo em relação aos dados de NDVI também foram relatadas por Povh (2007), evidenciando a necessidade de calibração específica para cada cultivar, uma vez que as diferenças de NDVI não foram explicadas nem pela MS acumulada e nem pelo teor de $\mathrm{N}$ foliar.
É notável que os resultados não se mantiveram os mesmos ao longo do ciclo de desenvolvimento da cultura, o que nas leituras em Feeks 3 pode ser explicado pela influência do ambiente, que pode interferir nos níveis de NDVI, pois nesse momento a cultura não apresenta biomassa suficiente para cobertura total do solo, ficando as leituras realizadas expostas tanto ao solo descoberto como a restos culturais de culturas antecessoras. Para as leituras realizadas em Feeks 10.5.1, os níveis de NDVI sofrem forte influência da presença de espigas e aristas na cultura, para ambos os sensores, resultando em valores de NDVI menores, comparados à avaliação em Feeks 8. Estudos realizados por Galvão et al. (1999), Grohs et al. (2009) e Flowers et al. (2003) também revelaram a influência de variáveis da planta e ambiente nas leituras de NDVI. 
Leituras de NDVI e as variáveis MS e teor foliar de $\mathrm{N}$

A elevação das doses de $\mathrm{N}$ proporcionou incremento na produção de MS, e como o aumento de MS implica em aumento de reflectância no infravermelho, os níveis de NDVI também aumentaram. Indiferentemente do sensor utilizado, o NDVI deve ser maior em situações onde há maior quantidade de MS (Serrano et al., 2000).

Neste trabalho, quando o NDVI foi utilizado na estimativa da MS (regressão), foram encontrados coeficientes de determinação $\left(R^{2}\right)$ superiores a 0,85 para o sensor passivo. Para o sensor ativo, os coeficientes foram superiores a 0,81 (Quadro 3). Ao realizar um estudo de leituras de NDVI com doses de $0,30,60,90$ e $120 \mathrm{~kg} \mathrm{ha}^{-1}$ de $\mathrm{N}$ na cultura do trigo, Povh (2007) também encontrou valores de $R^{2}$ superiores a 0,83 entre NDVI e MS.

Embora os resultados tenham comprovado a relação entre MS e NDVI para todos os estádios fenológicos avaliados, em Feeks 8 foram encontrados os maiores valores de $\mathrm{R}^{2}$ para todas as cultivares e para os dois sensores (Quadro 3). Isso ocorre em razão de a cultura, nesse momento, apresentar bom aporte de biomassa de parte aérea e cobertura do solo, e ainda estar sem as espigas e aristas, que influenciam negativamente as leituras de NDVI.

Observou-se regressão entre NDVI e teor foliar de N, para leituras realizadas com o sensor passivo, em dois agrupamentos: um com coeficientes

Quadro 3. Equações ajustadas entre a matéria seca (MS) da parte aérea, em $\mathrm{g} \mathrm{m}^{-2}$, de quatro cultivares de trigo e os valores de índice de vegetação por diferença normalizada (NDVI), obtidos por sensores passivo e ativo, em três estádios fenológicos da cultura submetida e cinco doses de nitrogênio (0 a $\left.120 \mathrm{~kg} \mathrm{ha}^{-1}\right)$

\begin{tabular}{|c|c|c|c|}
\hline Cultivar & Estádio fenológico & Equação & $\mathbf{R}^{2}$ \\
\hline \multicolumn{4}{|c|}{ Sensor passivo } \\
\hline Quartzo & Feeks 3 & $\mathrm{MS}=-0,361+0,0354 \mathrm{NDVI}-0,0006 \mathrm{NDVI}^{2}$ & $0,87^{* *}$ \\
\hline Campeiro & Feeks 3 & $\mathrm{MS}=-0,1194+0,0152 \mathrm{NDVI}-0,0002 \mathrm{NDVI}^{2}$ & $0,93^{* *}$ \\
\hline Tangara & Feeks 3 & $\mathrm{MS}=-0,1613+0,02 \mathrm{NDVI}-0,0002 \mathrm{NDVI}^{2}$ & $0,85^{* *}$ \\
\hline CD 115 & Feeks 3 & $\mathrm{MS}=-0,2944+0,0274 \mathrm{NDVI}-0,0004 \mathrm{NDVI}^{2}$ & $0,97^{* *}$ \\
\hline Quartzo & Feeks 8 & $\mathrm{MS}=0,0585+0,0014 \mathrm{NDVI}-0,0000008 \mathrm{NDVI}^{2}$ & $0,98^{* *}$ \\
\hline Campeiro & Feeks 8 & $\mathrm{MS}=-0,2802+0,0047 \mathrm{NDVI}-0,000008 \mathrm{NDVI}^{2}$ & $0,98^{* *}$ \\
\hline Tangara & Feeks 8 & $\mathrm{MS}=-0,3659+0,005 \mathrm{NDVI}-0,000007 \mathrm{NDVI}^{2}$ & $0,97^{* *}$ \\
\hline CD 115 & Feeks 8 & $\mathrm{MS}=0,0447+0,0022 \mathrm{NDVI}-0,000003 \mathrm{NDVI}^{2}$ & $0,99^{* *}$ \\
\hline Quartzo & Feeks 10.5.1 & $\mathrm{MS}=0,4927-0,0007 \mathrm{NDVI}+0,0000007 \mathrm{NDVI}^{2}$ & $0,93^{* *}$ \\
\hline Campeiro & Feeks 10.5.1 & $\mathrm{MS}=-2,3354+0,0062 \mathrm{NDVI}-0,000004 \mathrm{NDVI}^{2}$ & $0,92^{* *}$ \\
\hline Tangara & Feeks 10.5.1 & $\mathrm{MS}=-3,9778+0,0105 \mathrm{NDVI}-0,000006 \mathrm{NDVI}^{2}$ & $0,88^{* *}$ \\
\hline \multirow[t]{2}{*}{ CD 115} & Feeks 10.5.1 & $\mathrm{MS}=-0,2444+0,0013 \mathrm{NDVI}-0,0000006 \mathrm{NDVI}^{2}$ & $0,95^{* *}$ \\
\hline & \multicolumn{3}{|c|}{ Sensor ativo } \\
\hline Quartzo & Feeks 3 & $\mathrm{MS}=0,2099+0,0175$ NDVI- 0,0003 $\mathrm{NDVI}^{2}$ & $0,95^{* *}$ \\
\hline Campeiro & Feeks 3 & $\mathrm{MS}=0,339+0,0048$ NDVI- $0,00006 \mathrm{NDVI}^{2}$ & $0,87^{* *}$ \\
\hline Tangara & Feeks 3 & $\mathrm{MS}=0,3384+0,0067 \mathrm{NDVI}-0,00008 \mathrm{NDVI}^{2}$ & $0,81^{* *}$ \\
\hline CD 115 & Feeks 3 & $\mathrm{MS}=0,1659+0,0178 \mathrm{NDVI}-0,0003 \mathrm{NDVI}^{2}$ & $0,98^{* *}$ \\
\hline Quartzo & Feeks 8 & $\mathrm{MS}=0,5508+0,0006 \mathrm{NDVI}-0,0000003 \mathrm{NDVI}^{2}$ & $0,96^{* *}$ \\
\hline Campeiro & Feeks 8 & $\mathrm{MS}=0,4142+0,0019 \mathrm{NDVI}-0,000003 \mathrm{NDVI}^{2}$ & $0,93^{* *}$ \\
\hline Tangara & Feeks 8 & $\mathrm{MS}=0,5246+0,001 \mathrm{NDVI}-0,000009 \mathrm{NDVI}^{2}$ & $0,97^{* *}$ \\
\hline CD 115 & Feeks 8 & $\mathrm{MS}=0,6101+0,0006 \mathrm{NDVI}-0,0000003 \mathrm{NDVI}^{2}$ & $0,99^{* *}$ \\
\hline Quartzo & Feeks 10.5.1 & $\mathrm{MS}=0,8598-0,0006 \mathrm{NDVI}+0,0000005 \mathrm{NDVI}^{2}$ & $0,92^{* *}$ \\
\hline Campeiro & Feeks 10.5.1 & $\mathrm{MS}=-0,4124+0,0024 \mathrm{NDVI}-0,000001 \mathrm{NDVI}^{2}$ & $0,93^{* *}$ \\
\hline Tangara & Feeks 10.5.1 & $\mathrm{MS}=-1,8766+0,0062 \mathrm{NDVI}-0,000004 \mathrm{NDVI}^{2}$ & $0,83^{* *}$ \\
\hline CD 115 & Feeks 10.5.1 & $\mathrm{MS}=0,2846+0,0008 \mathrm{NDVI}-0,0000004 \mathrm{NDVI}^{2}$ & $0,99^{* *}$ \\
\hline
\end{tabular}

**: significativo a $1 \%$ pelo teste $\mathrm{F}$. 

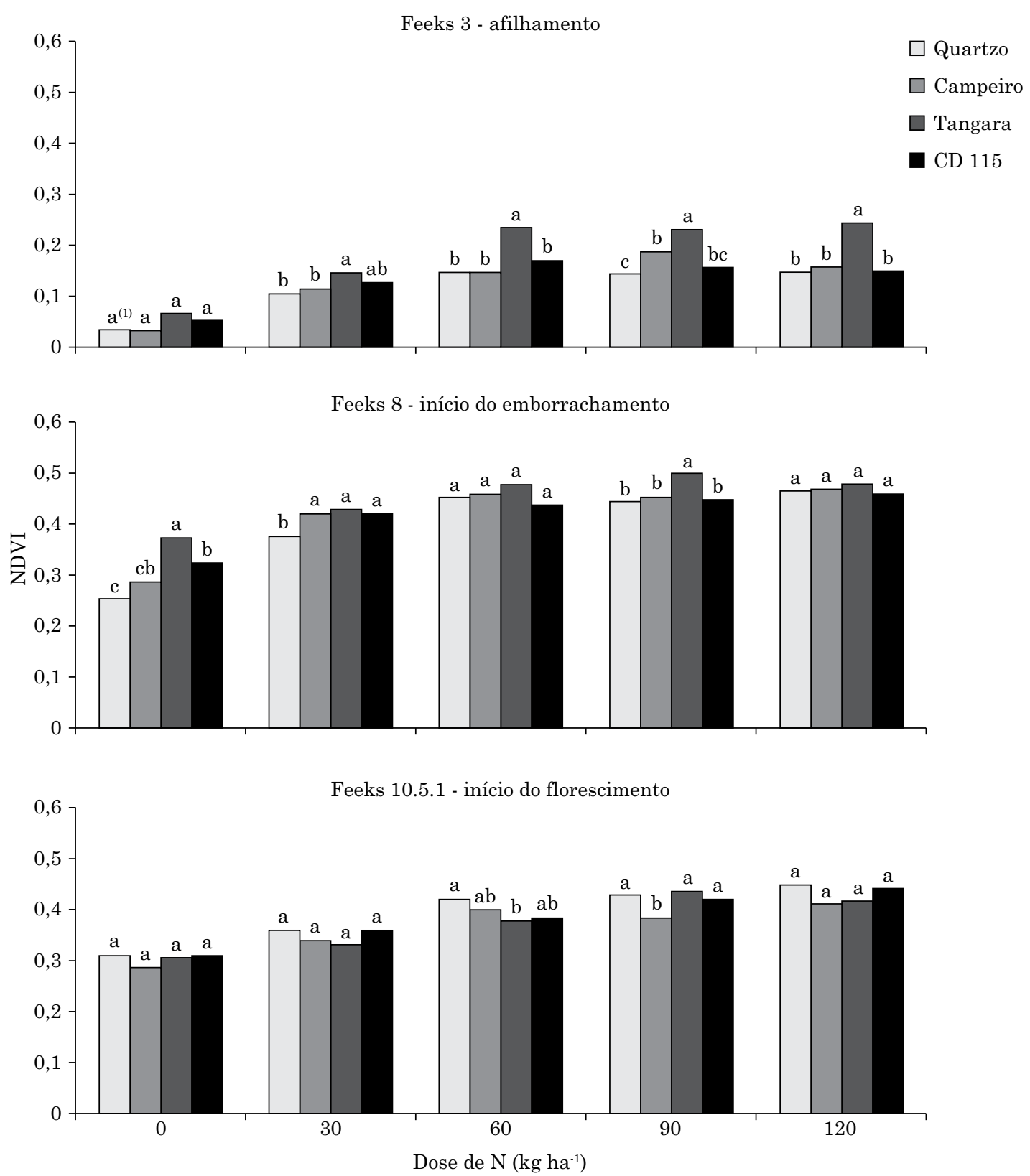

Figura 1. Índices de vegetação por diferença normalizada (NDVI) obtidos pelo sensor passivo, das cultivares de trigo em razão das doses de nitrogênio $(\mathrm{N})$, nos distintos estádios fenológicos. Letras comparam cultivares para cada dose de $\mathrm{N}$, em cada estádio fenológico, pelo teste Tukey a $5 \%$.

altamente significativos e elevados, superiores a 0,90, para 'Tangara' e 'CD 115', nos três estádios fenológicos; e outro com coeficientes inferiores a 0,90, para 'Quartzo' e 'Campeiro' (Quadro 4). Para o sensor ativo, os resultados indicaram situação semelhante, em que 'Quartzo' e 'Campeiro' apresentaram valores de $\mathrm{R}^{2}$ inferiores às demais cultivares ao longo do ciclo, reforçando a ideia de que diferenças genotípicas entre cultivares interferem na capacidade de concentrar o $\mathrm{N}$ na folha bandeira, bem como nas leituras dos sensores. Com o sensor ativo, $R^{2}$ baixos não significativos foram encontrados entre NDVI e teor foliar de $\mathrm{N}$ para 'Quartzo' e 'Campeiro', indicando que no estádio fenológico Feeks 3 ainda não havia características próprias desses materiais com relação determinada nas leituras de NDVI.

Neste trabalho, os resultados de relações entre NDVI e teor foliar de $\mathrm{N}$ foram superiores aos encontrados por Wright (2004), o qual em regressão de NDVI com teor foliar de $\mathrm{N}$ encontrou $\mathrm{R}^{2}$ de 0,66. Em leituras de NDVI nos estádios fenológicos Feeks 9 e Feeks 10.5, Freeman 

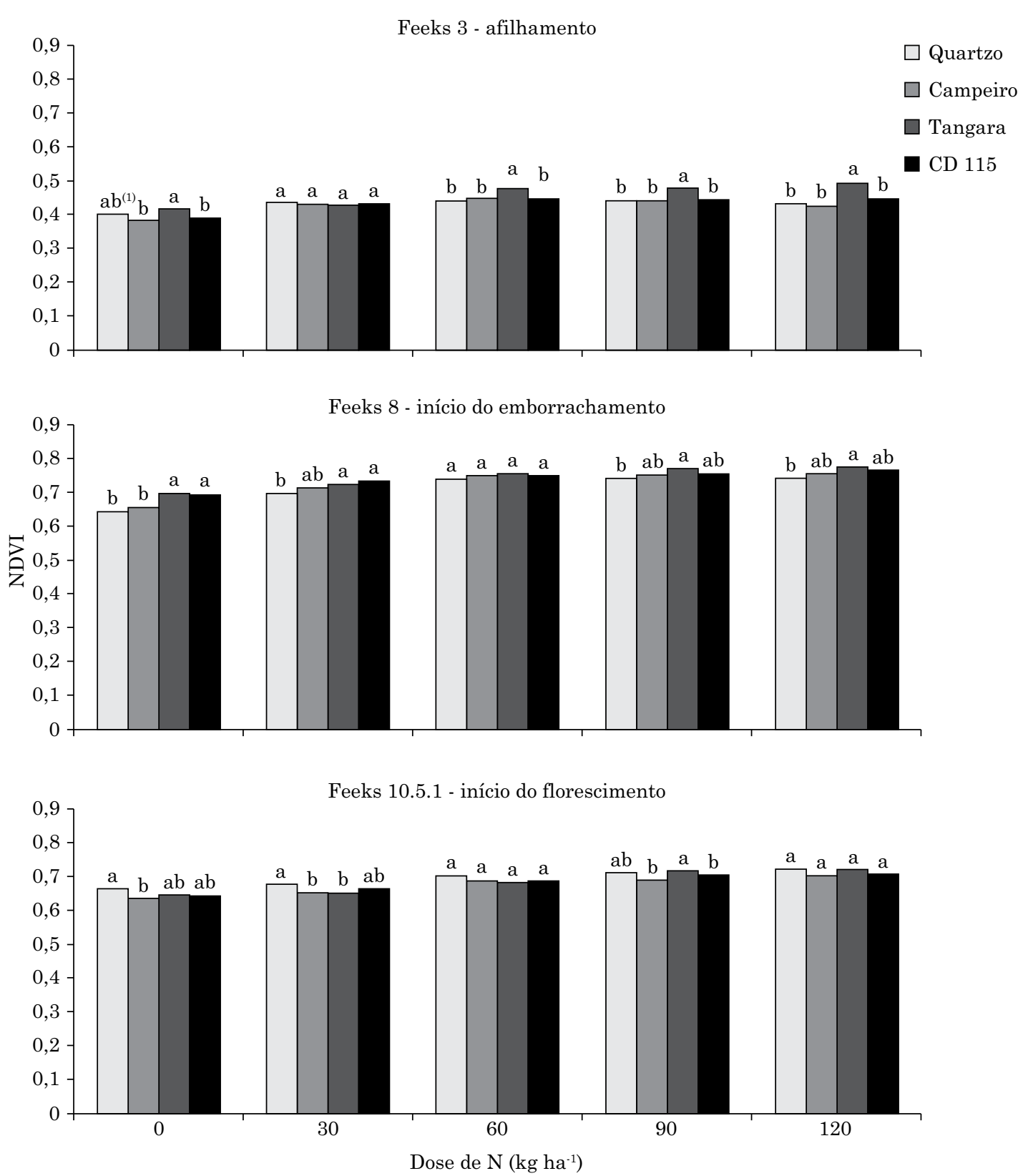

Figura 2. Índices de vegetação por diferença normalizada (NDVI) obtidos pelo sensor ativo, das cultivares de trigo em razão das doses de nitrogênio $(\mathrm{N})$, nos distintos estádios fenológicos. Letras comparam cultivares para cada dose de $\mathrm{N}$, em cada estádio fenológico, pelo teste Tukey a $5 \%$.

et al. (2003) chegaram a $\mathrm{R}^{2}$ de 0,57 e 0,48 respectivamente, estudando mais de 10 locais diferentes em duas safras. Em Feeks 6, Moges et al. (2004) encontraram $\mathrm{R}^{2}$ próximos de 0,91 , semelhante aos resultados para ambos os sensores no estádio Feeks 8 deste estudo.

\section{NDVI para estimativa de produtividade}

Com ambos os sensores e em todos os estádios fenológicos avaliados, obtiveram-se entre NDVI e produtividade, para cada cultivar, $\mathrm{R}^{2}$ superiores a
0,90 (Quadro 5) e altamente significativos ( $\mathrm{p}<0,01)$, confirmando a possibilidade de se estimar o potencial produtivo do trigo por meio do NDVI, ainda em estádios fenológicos em que a cultura está definindo o número de afilhos (Feeks 3). Encontraram-se resultados semelhantes em estudos realizados com técnicas de sensoriamento remoto, como o de Raun et al. (2002), que analisando diversos genótipos de trigo, geraram um algoritmo de estimativa de produtividade. Povh (2007) também constatou ser possível gerar modelos de estimativa de potencial produtivo de trigo, usando a relação de NDVI e 
Quadro 4. Equações ajustadas entre teor foliar de nitrogênio $(\mathrm{N})$, em $\mathrm{g} \mathrm{kg}^{-1}$, de quatro cultivares de trigo e os valores de índice de vegetação por diferença normalizada (NDVI), obtidos por sensores passivo e ativo, em três estádios fenológicos da cultura submetida a cinco doses de nitrogênio $\left(0\right.$ a $\left.120 \mathrm{~kg} \mathrm{ha}^{-1}\right)$

\begin{tabular}{|c|c|c|c|}
\hline Cultivar & Estádio fenológico & Equação & $\mathrm{R}^{2}$ \\
\hline & & Sensor passivo & \\
\hline Quartzo & Feeks 3 & $\mathrm{~N}=-17,619+0,8685 \mathrm{NDVI}-0,0106 \mathrm{NDVI}^{2}$ & $0,78^{* *}$ \\
\hline Campeiro & Feeks 3 & $\mathrm{~N}=-40,804+1,9667 \mathrm{NDVI}-0,0236 \mathrm{NDVI}^{2}$ & $0,70^{* *}$ \\
\hline Tangara & Feeks 3 & $\mathrm{~N}=-6,3056+0,3112 \mathrm{NDVI}-0,0037 \mathrm{NDVI}^{2}$ & $0,91^{* *}$ \\
\hline CD 115 & Feeks 3 & $\mathrm{~N}=-3,6213+0,1902 \mathrm{NDVI}-0,0024 \mathrm{NDVI}^{2}$ & $0,98^{* *}$ \\
\hline Quartzo & Feeks 8 & $\mathrm{~N}=-29,006+1,4350 \mathrm{NDVI}-0,0175 \mathrm{NDVI}^{2}$ & $0,79^{* *}$ \\
\hline Campeiro & Feeks 8 & $\mathrm{~N}=-35,454+1,7129 \mathrm{NDVI}-0,0204 \mathrm{NDVI}^{2}$ & $0,73^{* *}$ \\
\hline Tangara & Feeks 8 & $\mathrm{~N}=-4,4843+0,2388 \mathrm{NDVI}-0,0029 \mathrm{NDVI}^{2}$ & $0,94^{* *}$ \\
\hline CD 115 & Feeks 8 & $\mathrm{~N}=-3,0288+0,1707 \mathrm{NDVI}-0,0021 \mathrm{NDVI}^{2}$ & $0,97^{* *}$ \\
\hline Quartzo & Feeks 10.5.1 & $\mathrm{N}=-16,321+0,8094 \mathrm{NDVI}-0,0098 \mathrm{NDVI}^{2}$ & $0,89^{* *}$ \\
\hline Campeiro & Feeks 10.5.1 & $\mathrm{N}=-26,568+1,2815 \mathrm{NDVI}-0,0152 \mathrm{NDVI}^{2}$ & $0,74^{* *}$ \\
\hline Tangara & Feeks 10.5.1 & $\mathrm{N}=-1,5037+0,0798 \mathrm{NDVI}-0,0008 \mathrm{NDVI}^{2}$ & $0,90^{* *}$ \\
\hline \multirow[t]{2}{*}{ CD 115} & Feeks 10.5.1 & $\mathrm{N}=0,6101-0,0272 \mathrm{NDVI}+0,0005 \mathrm{NDVI}^{2}$ & $0,97^{* *}$ \\
\hline & & Sensor ativo & \\
\hline Quartzo & Feeks 3 & - & ns \\
\hline Campeiro & Feeks 3 & - & ns \\
\hline Tangara & Feeks 3 & $\mathrm{~N}=-0,6456+0,0471 \mathrm{NDVI}-0,0005 \mathrm{NDVI}^{2}$ & $0,83^{* *}$ \\
\hline CD 115 & Feeks 3 & $\mathrm{~N}=-1,4692+0,0958 \mathrm{NDVI}-0,0012 \mathrm{NDVI}^{2}$ & $0,99^{* *}$ \\
\hline Quartzo & Feeks 8 & $\mathrm{~N}=-16,113+0,8246 \mathrm{NDVI}-0,0101 \mathrm{NDVI}^{2}$ & $0,82^{* *}$ \\
\hline Campeiro & Feeks 8 & $\mathrm{~N}=-24,142+1,1896 \mathrm{NDVI}-0,0142 \mathrm{NDVI}^{2}$ & $0,71^{* *}$ \\
\hline Tangara & Feeks 8 & $\mathrm{~N}=-0,9338+0,0763 \mathrm{NDVI}-0,0009 \mathrm{NDVI}^{2}$ & $0,95^{* *}$ \\
\hline CD 115 & Feeks 8 & $\mathrm{~N}=-0,4541+0,0561 \mathrm{NDVI}-0,0006 \mathrm{NDVI}^{2}$ & $0,96^{* *}$ \\
\hline Quartzo & Feeks 10.5 .1 & $\mathrm{~N}=-4,8881+0,2673 \mathrm{NDVI}-0,0032 \mathrm{NDVI}^{2}$ & $0,89^{* *}$ \\
\hline Campeiro & Feeks 10.5.1 & $\mathrm{N}=-14,323+0,712 \mathrm{NDVI}-0,0084 \mathrm{NDVI}^{2}$ & $0,92^{* *}$ \\
\hline Tangara & Feeks 10.5.1 & $\mathrm{N}=0,7831-0,0156 \mathrm{NDVI}+0,0003 \mathrm{NDVI}^{2}$ & $0,92^{* *}$ \\
\hline CD 115 & Feeks 10.5.1 & $\mathrm{N}=0,7398-0,0112 \mathrm{NDVI}+0,0003 \mathrm{NDVI}^{2}$ & $0,99^{* *}$ \\
\hline
\end{tabular}

** $\mathrm{e}^{\mathrm{ns}}$ : significativo a $1 \%$ e não significativo pelo teste $\mathrm{F}$, respectivamente.

produtividade, com elevado nível de confiança, sendo também constatado por Inman et al. (2005) e Freeman et al. (2003).

O NDVI pode ser utilizado como indicador de potencial produtivo, quando obtido no momento de maior acúmulo de biomassa (Liu, 2006). No entanto, vale ressaltar que quando a estimativa de produtividade ocorre em estádios fenológicos, em que a cultura ainda encontra-se em desenvolvimento inicial, esse resultado pode ser comprometido por efeito de fatores biótico e abióticos sobre as plantas, como doenças, pragas e condições meteorológicas, fatos não observados neste estudo, onde leituras de NDVI em Feeks 3 resultaram em alta relação com a produtividade final de grãos. Portanto, o modelo de estimativa de potencial produtivo é viável se o NDVI apresentar alta relação com a variável indicadora do status nitrogenado no dossel da cultura, e que esse, por sua vez, não sofra interferência de outros fatores.

No presente estudo, foi possível gerar modelos de estimativa de produtividade de grãos de trigo para a região de Guarapuava, PR, com base na reflectância do dossel, para todas as cultivares analisadas, o que, no futuro, pode abrir a possibilidade de subsidiar a aplicação de doses de $\mathrm{N}$ em taxa variável, podendo, inclusive, se aprimoradas as técnicas de leitura e aplicação do fertilizante em termos de equipamentos, realizar a variação da dose de $\mathrm{N}$ em tempo real. 
Quadro 5. Produtividade de trigo (PROD), em $\mathrm{kg} \mathrm{ha}^{-1}$, de quatro cultivares de trigo e os valores de índice de vegetação por diferença normalizada (NDVI), obtidos por sensores passivo e ativo, em três estádios fenológicos da cultura submetida a cinco doses de nitrogênio (0 a $\left.120 \mathrm{~kg} \mathrm{ha}^{-1}\right)$

\begin{tabular}{|c|c|c|c|}
\hline Cultivar & Estádio fenológico & Equação & $\mathbf{R}^{2}$ \\
\hline & & Sensor passivo & \\
\hline Quartzo & Feeks 3 & $\mathrm{PROD}=3.679,3+8.447,4 \mathrm{NDVI}+21.273 \mathrm{NDVI}^{2}$ & $0,96^{* *}$ \\
\hline Campeiro & Feeks 3 & $\mathrm{PROD}=3.490,0+15.705 \mathrm{NDVI}-30.126 \mathrm{NDVI}^{2}$ & $0,95^{* *}$ \\
\hline Tangara & Feeks 3 & $\mathrm{PROD}=4.172,4-3.555,7 \mathrm{NDVI}+24.847 \mathrm{NDVI}^{2}$ & $0,92^{* *}$ \\
\hline CD 115 & Feeks 3 & $\mathrm{PROD}=3.337,4+10.950 \mathrm{NDVI}-22.531 \mathrm{NDVI}^{2}$ & $0,95^{* *}$ \\
\hline Quartzo & Feeks 8 & $\mathrm{PROD}=3.033,7+2.209,4 \mathrm{NDVI}+6.473,2 \mathrm{NDVI}^{2}$ & $0,97^{* *}$ \\
\hline Campeiro & Feeks 8 & $\mathrm{PROD}=7.268,8-23.230 \mathrm{NDVI}+41.112 \mathrm{NDVI}^{2}$ & $0,99^{* *}$ \\
\hline Tangara & Feeks 8 & $\mathrm{PROD}=10.766,0-36.543 \mathrm{NDVI}+49.613 \mathrm{NDVI}^{2}$ & $0,95^{* *}$ \\
\hline CD 115 & Feeks 8 & $\mathrm{PROD}=11,6+16.814 \mathrm{NDVI}-15.197 \mathrm{NDVI}^{2}$ & $0,92^{* *}$ \\
\hline Quartzo & Feeks 10.5.1 & $\mathrm{PROD}=-4.695,3+40.416 \mathrm{NDVI}-39.500 \mathrm{NDVI}^{2}$ & $0,98^{* *}$ \\
\hline Campeiro & Feeks 10.5.1 & $\mathrm{PROD}=-3.208,5+36.181 \mathrm{NDVI}-37.337 \mathrm{NDVI}^{2}$ & $0,98^{* *}$ \\
\hline Tangara & Feeks 10.5.1 & PROD $=-279,4+19.636$ NDVI -18.113 NDVI $^{2}$ & $0,98^{* *}$ \\
\hline \multirow[t]{2}{*}{ CD 115} & Feeks 10.5.1 & $\mathrm{PROD}=-7.069,1+56.707 \mathrm{NDVI}-69.212 \mathrm{NDVI}^{2}$ & $0,97^{* *}$ \\
\hline & & Sensor ativo & \\
\hline Quartzo & Feeks 3 & $\mathrm{PROD}=-163.210,0+768.065 \mathrm{NDVI}-875.536 \mathrm{NDVI}^{2}$ & $0,91^{* *}$ \\
\hline Campeiro & Feeks 3 & $\mathrm{PROD}=-58.839,0+287.697 \mathrm{NDVI}-323.115 \mathrm{NDVI}^{2}$ & $0,90^{* *}$ \\
\hline Tangara & Feeks 3 & $\mathrm{PROD}=-21.466,0+104.440 \mathrm{NDVI}-103.936 \mathrm{NDVI}^{2}$ & $0,94^{* *}$ \\
\hline CD 115 & Feeks 3 & $\mathrm{PROD}=-1.689,7+16.845 \mathrm{NDVI}-6.565,2 \mathrm{NDVI}^{2}$ & $0,93^{* *}$ \\
\hline Quartzo & Feeks 8 & $\mathrm{PROD}=-7.308,4+20.904 \mathrm{NDVI}-5.145,4 \mathrm{NDVI}^{2}$ & $0,96^{* *}$ \\
\hline Campeiro & Feeks 8 & $\mathrm{PROD}=1.891,8-5.753,5 \mathrm{NDVI}+13.640 \mathrm{NDVI}^{2}$ & $0,99^{* *}$ \\
\hline Tangara & Feeks 8 & $\mathrm{PROD}=21.491,0-56.925 \mathrm{NDVI}+45.698 \mathrm{NDVI}^{2}$ & $0,94^{* *}$ \\
\hline CD 115 & Feeks 8 & $\mathrm{PROD}=-76.308,0+212.324 \mathrm{NDVI}-139.469 \mathrm{NDVI}^{2}$ & $0,94^{* *}$ \\
\hline Quartzo & Feeks 10.5 .1 & $\mathrm{PROD}=-184.088,0+521.271 \mathrm{NDVI}-358.337 \mathrm{NDVI}^{2}$ & $0,96^{* *}$ \\
\hline Campeiro & Feeks 10.5.1 & $\mathrm{PROD}=-161.574,0+478.251 \mathrm{NDVI}-342.608 \mathrm{NDVI}^{2}$ & $0,97^{* *}$ \\
\hline Tangara & Feeks 10.5.1 & $\mathrm{PROD}=-73.058,0+217.086 \mathrm{NDVI}-151.352 \mathrm{NDVI}^{2}$ & $0,99^{* *}$ \\
\hline CD 115 & Feeks 10.5.1 & $\mathrm{PROD}=-120.710,0+359.868 \mathrm{NDVI}-258.477 \mathrm{NDVI}^{2}$ & $0,95^{* *}$ \\
\hline
\end{tabular}

**: significativo a $1 \%$ pelo teste $\mathrm{F}$.

\section{CONCLUSÕES}

As leituras de NDVI obtidas para as cultivares, com ambos os sensores, foram influenciadas pelas doses de $\mathrm{N}$ e estádios fenológicos do trigo.

O NDVI interfere nas variáveis MS da parte aérea e teor foliar de $\mathrm{N}$ do trigo para todas as cultivares estudadas. Consequentemente, o NDVI obtido por ambos os sensores apresentou relação direta com a produtividade para os três estádios fenológicos avaliados, possibilitando a elaboração de modelos de estimativa de produtividade desde o início de desenvolvimento da cultura.

\section{REFERÊNCIAS}

Cantarella H, Raij Bvan, Camargo CEO. Cereais. In: Raij Bvan; Cantarella H, Quaggio JA, Furlani AMC, editores. Recomendações de calagem e adubação para o Estado de São Paulo. Campinas: Instituto Agronômico de Campinas; 1997. (Boletim técnico, 100).

Empresa Brasileira de Pesquisa Agropecuária - Embrapa. Centro Nacional de Pesquisa de Solos. Sistema brasileiro de classificação de solos. $2^{\mathrm{a}}$. ed. Rio de Janeiro: 2006.

Ernani PR. Disponibilidade de nitrogênio e adubação nitrogenada para macieira. Lages: Graphel; 2003.

Food and Agricultural Organization of the United Nations FAO. Faostat. [Acessado em: 17 abr. 2015]. Disponível em: http://faostat3.fao.org/download/Q/QC/E. 
Flowers MR, Weisz R, Heiniger B,Tarleton AM. Field validation of a remote sensing technique for early nitrogen application decisions in wheat. Agron J. 2003;95:167-76.

Freeman KW, Raun WR, Johnson GV, Mullen RW, Stone ML, Solie JB. Late-season prediction of wheat grain yield and grain protein. Commun Soil Sci Plant Anal. 2003;34:1837-52.

Galvão LS, Vitorello I, Almeida Filho R. Effects of band positioning and bandwidth on NDVI measurements of tropical savannas. Rem Sens Environ. 1999;67:181-93.

Gastal F, Lemaire GN. Uptake and distribution in crops: an agronomical and ecophysiological perspective. J Exper Bot. 2002;53:789-99.

Grohs DS, Bredemeier C, Mundstock CM, Poletto N. Modelo para estimativa do potencial produtivo em trigo e cevada por meio do sensor GreenSeeker. Eng Agríc. 2009;29:101-12.

Hatfield JL. Spectral behavior of wheat yield variety trials. Photogramm Eng Rem Sensing. 1981;47:1487-91.

Inman D, Khosla R, Westfall DG. Nitrogen uptake across site specific management zones in irrigated maize production systems. Agron J. 2005;2005;97:169-76.

Instituto Brasileiro de Geografia e Estatística - IBGE. Sistema IBGE de recuperação automática - Sidra. [Acessado em: $17 \mathrm{abr}$. 2015]. Disponível em: http://www.sidra.ibge.gov.br/bda/tabela/ protabl.asp?c $=1612 \& \mathrm{z}=\mathrm{t} \& \mathrm{o}=10 \& \mathrm{i}=\mathrm{P}$.

Large EC. Growth stages in cereals illustration of the Feeks scales. Plant Pathol. 1954;4:22-4.

Liu WTH. Aplicações de sensoriamento remoto. Campo Grande: UNIDERP; 2006.

Moges SM, Raun WR, Mullen RW, Freeman KW, Johnson GV, Solie JB. Evaluation of green, red, and near infrared bands for predicting winter wheat biomass, nitrogen uptake, and final grain yield. J Plant Nutr. 2004;27:1431-41.
Pavan MA, Bloch MF, Zempulski HC, Miyazawa M, Zocoler DC. Manual de análise química de solo e controle de qualidade. Londrina: Iapar; 1992.

Ponzoni FJ. Comportamento espectral da vegetação. In. Meneses PR, Madeira Netto JS, editores. Sensoriamento remoto reflectância dos alvos naturais. Brasília, DF: Universidade de Brasília; 2001. p.157-99.

Povh FP. Utilização de sensor ótico ativo em culturas de cereais [dissertação]. Piracicaba: Escola Superior de Agricultura "Luiz de Queiroz"; 2007.

Raun WR, Solie JB, Johnson GV, Stone ML, Mullen RW, Freeman KW, Thomason WE, Lukina EV. Improving nitrogen use efficiency in cereal grain production with optical sensing and variable rate application. Agron J. 2002;94:815-20.

Rouse JW, Haas RH, Schell JA, Deering DW. Monitoring vegetation systems in the Great Plains with ERTS. In: Proceedings of the $3^{\text {rd }}$. ERTS-1 Symposium; 1973; Washington, DC. Washington, DC: NASA SP-351; 1973. p.309-17.

Schadchina TM, Dmitrieva VV. Leaf chlorophyll content as a possible diagnostic mean for the evaluation of plant nitrogen uptake from the soil. J Plant Nutr. 1995;18:1427-37.

Serrano L, Filella I, Peñuelas J. Remote sensing of biomass and yield of winter wheat under different nitrogen supplies. Crop Sci. 2000;40:723-31.

Tucker CJ. Red and photographic infrared linear combination for monitoring vegetation. Rem Sens Environ. 1979;8:127-50.

Wright DL. Canopy reflectance estimation of wheat nitrogen content for grain protein management. GISci Rem Sens. 2004;41:287-300.

Zagonel J, Venâncio WS, Kunz RP, Tanamati H. Doses de nitrogênio e densidade de plantas com e sem um regulador de crescimento afetando o trigo, cultivar OR-1. Ci Rural. 2002;32:25-9. 